PoS $\quad \begin{aligned} & \text { PROCEEDINGS } \\ & \text { OF SCIENCE }\end{aligned}$

\title{
Higgs bosons in the Next-to-Minimal Supersymmetric Standard Model
}

\section{Ulrich ELLWANGER*}

LPT Orsay, UMR 8627, CNRS, Université de Paris-Sud, 91405 Orsay

E-mail: ellwanger@th.u-psud.fr

We discuss how decays of Higgs bosons into light pseudoscalars $A_{1}$ reduce the required fine tuning in the parameter space of the constrained NMSSM as compared to the MSSM, notably for $M_{A_{1}} \sim 10 \mathrm{GeV}$ where $A_{1}-\eta_{b}$ mixing is relevant. Due to the induced dominant $A_{1} \rightarrow g g$ decays, the search for jet substructures seems to be the only hope for Higgs discovery at the LHC in such a scenario.

XXIst International Europhysics Conference on High Energy Physics

21-27 July 2011

Grenoble, Rhônes Alpes France

${ }^{*}$ Speaker. 
The first motivation for supersymmetric extensions of the Standard Model (SM) stems from the solution of the naturalness or finetuning problem in the Higgs sector of the SM. Since LEP has established a lower bound of about $114 \mathrm{GeV}$ on the mass $M_{h}$ of a SM-like Higgs boson, it has become clear that the Minimal Supersymmetric Standard Model (MSSM) suffers from a so-called "little hierarchy problem".

The origin of the little hierarchy problem can be understood as follows. The Lagrangian of the MSSM in the Higgs sector contains two doublets $H_{u}$ and $H_{d}$, soft supersymmetry (Susy) breaking mass terms $m_{H_{u}}^{2}$ and $m_{H_{d}}^{2}$ for these scalars, additional supersymmetric mass terms $\mu^{2}$ (whose origin is difficult to understand), and quartic couplings depending on the electroweak gauge couplings $g_{1}$ and $g_{2}$. In the approximation $\left\langle H_{u}\right\rangle \gg\left\langle H_{d}\right\rangle$ (neglecting terms $\sim \tan ^{-2} \beta$ ), the tree level potential $V$ reads simply

$$
V \simeq\left(m_{H_{u}}^{2}+\mu^{2}\right)\left|H_{u}\right|^{2}+\frac{g_{1}^{2}+g_{2}^{2}}{8}\left|H_{u}\right|^{4} .
$$

From $M_{Z}^{2}=\left\langle H_{u}^{2}\right\rangle \frac{g_{1}^{2}+g_{2}^{2}}{2}$ we find the condition

$$
-2\left(m_{H_{u}}^{2}+\mu^{2}\right) \stackrel{!}{=} M_{Z}^{2}
$$

In the absence of any finetuning, we should have $M_{Z}^{2} \approx \mu^{2} \approx-m_{H_{u}}^{2}$. However, the SM-like Higgs mass $M_{h}$ is approximately given by

$$
M_{h}^{2} \sim M_{Z}^{2}+\frac{3 m_{\text {top }}^{2}}{4 \pi^{2}\left\langle H_{u}\right\rangle^{2}} \ln \left(\frac{M_{\text {stop }}^{2}}{m_{\text {top }}^{2}}\right)+\ldots
$$

For $M_{h} \gtrsim 114 \mathrm{GeV}$ we need $M_{\text {stop }} \gtrsim 1 \mathrm{TeV}$, and but large values for $M_{\text {stop }}$ induce $m_{H_{u}}^{2} \sim-M_{\text {stop }}^{2}$ via radiative corrections between the weak and the GUT scale. $m_{H_{u}}^{2} \sim 1 \mathrm{TeV}^{2}$ requires to tune $\mu^{2}$ in Eq. (2) with a precision of $\approx 1 \%$.

In the Next-to-Minimal Supersymmetric Standard Model (NMSSM) [1], an additional gauge singlet superfield $S$ generates an effective $\mu_{e f f}$-term through its vacuum expectation value, $\mu_{e f f}=$ $\lambda\langle S\rangle$, where $\lambda$ is a Yukawa coupling. Apart from generating automatically a $\mu$-term of the desired order, the NMSSM has more physical states in the (neutral) Higgs sector as the MSSM: 3 neutral $\mathrm{CP}$-even, and two neutral CP-odd states. The lightest $\mathrm{CP}$-odd state $A_{1}$ can be quite light $(0<$ $M_{A_{1}} \lesssim 50 \mathrm{GeV}$ ) without contradicting any bounds. In this case the SM-like Higgs boson $h$ would decay dominantly as $h \rightarrow A_{1} A_{1}$, and LEP constraints on $M_{h}$ are alleviated: essentially one is left with constraints on $h \rightarrow 4 b$ (if $M_{A_{1}} \gtrsim 10.5 \mathrm{GeV}$ ) from DELPHI/OPAL [2, 3], and on $h \rightarrow 4 \tau$ (if $M_{A_{1}} \lesssim 10.5 \mathrm{GeV}$ ) from ALEPH [4].

The region $9.5 \mathrm{GeV} \lesssim M_{A_{1}} \lesssim 10.5 \mathrm{GeV}$ is particularly interesting: here $A_{1}$ would mix with the CP-odd $b \bar{b}$ bound states $\eta_{b}(n S)$. The mass of the only observed state $\eta_{b}(1 S)$ by BaBar $[5,6]$ is actually somewhat below expectations from QCD for the hyperfine splitting $M_{\Upsilon(1 S)}-M_{\eta_{b}(1 S)}$. This could be explained by $A_{1}-\eta_{b}(1 S)$ mixing, if $M_{A_{1}}$ is in the above range [7]. However, the width for any decay $\eta_{b}(n S) \rightarrow g g$ is much larger than the width $A_{1} \rightarrow \tau^{+} \tau^{-}$. Consequently a tiny $A_{1}-\eta_{b}(n S)$ mixing angle suffices such that the physical eigenstate decays dominantly into $g g$ [8], and the ALEPH constraints do not apply.

In general, $h \rightarrow A_{1} A_{1}$ decays alleviate the lower bounds on $M_{h}$ from LEP and hence the little fine tuning problem $[9,10,11]$. Taking possible $A_{1}-\eta_{b}(n S)$ mixing into account, the corresponding remaining finetuning in the constraint NMSSM (cNMSSM) has been studied in [12] and 

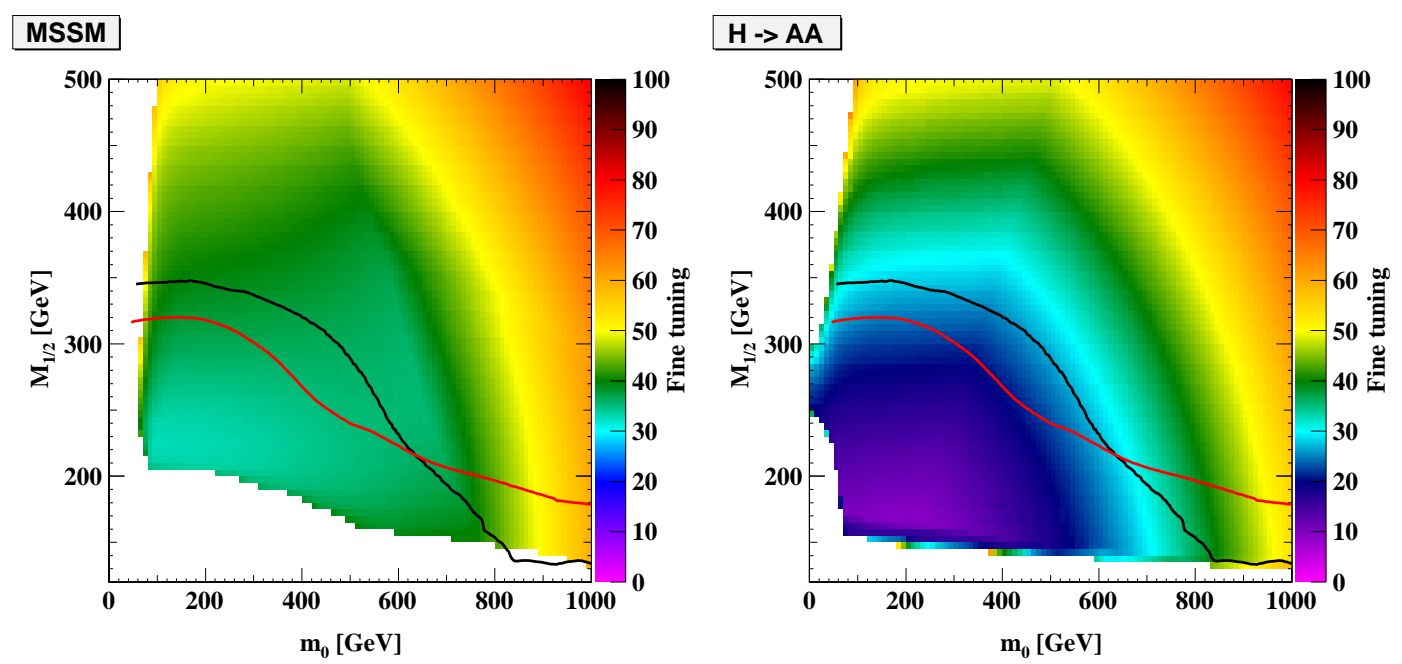

Figure 1: $\Delta$ in the plane $m_{0}-M_{1 / 2}$ for the cMSSM and the cNMSSM. Bounds within specific cMSSM scenarios from ATLAS [13] are indicated as black lines, and from CMS [14] as red lines.

compared to the cMSSM. Here, the finetuning measure is defined as

$$
\Delta=\operatorname{Max}\left\{\left|\frac{\partial \ln \left(M_{Z}\right)}{\partial \ln \left(p_{i}^{\mathrm{GUT}}\right)}\right|\right\}
$$

where $p_{i}^{\mathrm{GUT}}$ are the parameters at the GUT scale:

$$
p_{i}^{\mathrm{GUT}}=m_{0}, M_{1 / 2}, A_{0}, h_{t}, \ldots
$$

For fixed $m_{0}, M_{1 / 2}$ (universal scalar and gaugino masses) we look for the minimum of $\Delta$ as function of $A_{0}, \tan (\beta), \ldots$; the minimal value of $\Delta$ can be represented in the plane $m_{0}, M_{1 / 2}$ for the cMSSM and the cNMSSM: We see in Fig. 1 that, for $M_{1 / 2} \lesssim 400 \mathrm{GeV}$ and $m_{0} \lesssim 800 \mathrm{GeV}$, the amount of finetuning in the cNMSSM ( $\gtrsim 10$ ) can be considerably less than in the cMSSM ( $\gtrsim 33$ ) due to lower possible values of $M_{h}$ due to allowed $h \rightarrow A_{1} A_{1}$ decays, although most of this region is now excluded by fruitless searches for supersymmetry with low $M_{S u s y}$ at the LHC. (However, these negative results are not necessarily applicable to the cNMSSM, notably for a singlino-like LSP.)

It is interesting to study the dependence of the finetuning $\Delta$ on $M_{A_{1}}$ in the cNMSSM in Fig. 2. We see that $\Delta$ is particularly low for $M_{A_{1}} \sim 10 \mathrm{GeV}$ (where $h$ can be light due to the absence of constraints from $h \rightarrow 4 \tau$ ), and for $30 \mathrm{GeV} \lesssim M_{A_{1}} \lesssim 50 \mathrm{GeV}$ where the constraints on $M_{h}$ from $H \rightarrow 4 b$ are weak.

It is clear that, for any value of $M_{h}$ and $M_{A_{1}}$, the search for a SM-like Higgs boson decaying as $H \rightarrow A_{1} A_{1} \rightarrow \ldots$ is a challenge at the LHC (see [15] and references therein). Notably the interesting case $M_{A_{1}} \approx 10 \mathrm{GeV}$, where $A_{1} \rightarrow g g$ dominates, seems hopeless at first sight.

However, the search for jet substructures can be applied to such a situation [16, 17, 18, 19, 20]. Here one concentrates on associate $h$ production with a $W^{ \pm}$, and triggers on an isolated lepton from $W^{ \pm} \rightarrow l^{ \pm}+v$. Then one studies $h \rightarrow A_{1} A_{1} \rightarrow 2$ (fat) jets $j$ from each $A_{1}$. Typically one requires jet transverse momenta $p_{T_{j}}>100,50 \mathrm{GeV}$ (or $200 \mathrm{GeV}$ ) allowing to study a boosted Higgs. Then 


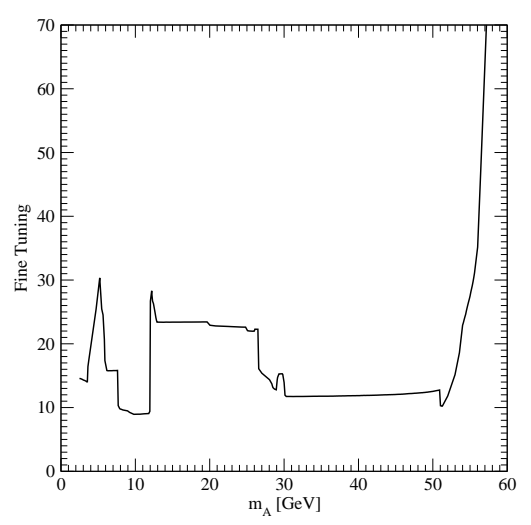

Figure 2: Dependence of the finetuning $\Delta$ on $M_{A_{1}}$ in the cNMSSM

one looks for substructures in jets $j$ with $m_{j} \lesssim 12 \mathrm{GeV}$, which are supposed to originate from an $A_{1}$ decay into 2 gluons: Undoing the last recombination step of the clustering algorithm from $j_{1}, j_{2}$ to $j$, one requires $m_{j_{1}} \sim m_{j_{2}} \ll m_{j}$. Finally one looks for a peak in the dijet mass $m_{j j} \sim m_{h}$. A possible result (from [16], with $m_{h}=120 \mathrm{GeV}, 30 \mathrm{fb}^{-1}$ luminosity) is shown in Fig. 3. Hence, as also indicated in $[17,18,19,20]$, such a search seems feasible.

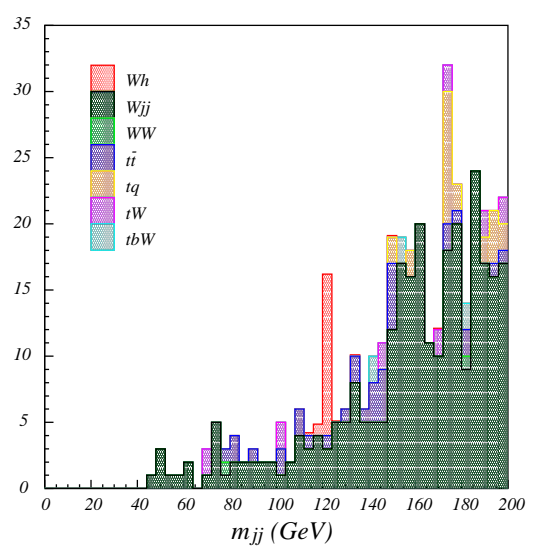

Figure 3: Possible result for the dijet mass $m_{j j}$ for $m_{h}=120 \mathrm{GeV}$, from [16]

To summarize, the scenario with a light pseudoscalar $A_{1}$ with $M_{A_{1}} \approx 10 \mathrm{GeV}$ in the NMSSM is particularly interesting, and particularly challenging: due to $A_{1}-\eta_{b}(1 S)$ mixing and the resulting dominant $A_{1} \rightarrow g g$ decays, bounds from LEP on $h \rightarrow A_{1} A_{1}$ are particularly weak. This allows for scenarios with particularly low finetuning in the (c)NMSSM. But, precisely this final state in Higgs decays is very difficult to detect. In this scenario, the search for jet substructures seems to be the only hope for a Higgs discovery at the LHC. 


\section{References}

[1] U. Ellwanger, C. Hugonie and A. M. Teixeira, Phys. Rept. 496 (2010) 1 [arXiv:0910.1785 [hep-ph]].

[2] G. Abbiendi et al. [OPAL Collaboration], Eur. Phys. J. C 37 (2004) 49 [arXiv:hep-ex/0406057].

[3] J. Abdallah et al. [DELPHI Collaboration], Eur. Phys. J. C 38 (2004) 1 [arXiv:hep-ex/0410017].

[4] S. Schael et al. [ALEPH Collaboration], JHEP 1005 (2010) 049 [arXiv:1003.0705].

[5] B. Aubert et al. [BABAR Collaboration], Phys. Rev. Lett. 101 (2008) 071801 [Erratum-ibid. 102 (2009) 029901] [arXiv:0807.1086 [hep-ex]].

[6] B. Aubert et al. [BABAR Collaboration], Phys. Rev. Lett. 103 (2009) 161801 [arXiv:0903.1124 [hep-ex]].

[7] F. Domingo, U. Ellwanger and M. A. Sanchis-Lozano, Phys. Rev. Lett. 103 (2009) 111802 [arXiv:0907.0348 [hep-ph]].

[8] F. Domingo and U. Ellwanger, JHEP 1106 (2011) 067 [arXiv:1105.1722 [hep-ph]].

[9] R. Dermisek and J. F. Gunion, Phys. Rev. Lett. 95 (2005) 041801 [arXiv:hep-ph/0502105].

[10] R. Dermisek and J. F. Gunion, Phys. Rev. D 73 (2006) 111701 [arXiv:hep-ph/0510322].

[11] R. Dermisek and J. F. Gunion, Phys. Rev. D 76 (2007) 095006 [arXiv:0705.4387 [hep-ph]].

[12] U. Ellwanger, G. Espitalier-Noel, C. Hugonie, "Naturalness and Fine Tuning in the NMSSM: Implications of Early LHC Results,” [arXiv:1107.2472 [hep-ph]], to appear in JHEP.

[13] ATLAS Collaboration, ATL-COM-PHYS-2011-981, presentation at the 2011 Europhysics Conference On High Energy Physics, Grenoble, France, 21 - 27 July 2011

[14] CMS Collaboration, "Search for supersymmetry in all-hadronic events with $\alpha_{T}$ ", CMS-PAS-SUS-11-003, presentation at the 2011 Europhysics Conference On High Energy Physics, Grenoble, France, 21 - 27 July 2011

[15] U. Ellwanger, "Higgs Bosons in the Next-to-Minimal Supersymmetric Standard Model at the LHC," [arXiv:1108.0157 [hep-ph]], to appear in EPJC

[16] C. R. Chen, M. M. Nojiri and W. Sreethawong, JHEP 1011 (2010) 012 [arXiv:1006.1151 [hep-ph]].

[17] A. Falkowski, D. Krohn, L. T. Wang, J. Shelton and A. Thalapillil, "Unburied Higgs," arXiv:1006.1650 [hep-ph].

[18] B. Bellazzini, C. Csaki, J. Hubisz and J. Shao, Phys. Rev. D 83 (2011) 095018 [arXiv:1012.1316 [hep-ph]].

[19] D. E. Kaplan and M. McEvoy, Phys. Rev. D 83 (2011) 115004 [arXiv:1102.0704 [hep-ph]].

[20] C. Englert, T. S. Roy and M. Spannowsky, “Ditau jets in Higgs searches,” arXiv:1106.4545 [hep-ph]. 